\title{
Evaluation of the usefulness of colonoscopy with mucosal biopsies in the follow-up of TNBS-induced colitis in rats
}

\author{
MAGDY EL-SALHY ${ }^{1,2}$, INGVILD HAUKAAS WENDELBO ${ }^{1,2}$, DORIS GUNDERSEN ${ }^{3}$, \\ JAN GUNNAR HATLEBAKK ${ }^{2}$ and TRYGVE HAUSKEN ${ }^{2}$ \\ ${ }^{1}$ Section for Gastroenterology, Department of Medicine, Stord Helse-Fonna Hospital, Stord; \\ ${ }^{2}$ Section for Gastroenterology, Institute of Medicine, University of Bergen, Bergen; \\ ${ }^{3}$ Department of Research, Helse-Fonna, Haugesund, Norway
}

Received January 24, 2013; Accepted May 29, 2013

DOI: $10.3892 / \mathrm{mmr} .2013 .1528$

\begin{abstract}
Animal models are required for research regarding the pathogenesis and efficacy of anti-inflammatory agents in inflammatory bowel disease (IBD). Trinitrobenzene sulfonic acid (TNBS)-induced colitis closely mimics Crohn's disease. The present study was undertaken in order to determine the reliability of following the inflammatory course of TNBSinduced colitis using colonoscopy together with biopsy samples obtained during the examination. In this study we used 20 adult male Wistar rats, with a mean weight of $201.9 \mathrm{~g}$. The rats were divided into two groups, control and TNBS, with ten rats in each group. Following the induction of TNBS colitis, the rats underwent colonoscopy with mucosal biopsies. At the end of the experiment, the rats were sacrificed and whole-wall colonic samples were obtained. The degree of inflammation was assessed endoscopically, macroscopically and microscopically. There was no significant change in the body weight of the control group but significant weight loss was observed in the TNBS group. Examination of the control group did not reveal any inflammation. Severe colitis was observed in the TNBS-induced colitis rats, as assessed endoscopically, macroscopically and microscopically. The endoscopic inflammation score obtained through colonoscopy examinations correlated with that obtained macroscopically, and those obtained microscopically from the whole-wall colon and biopsy samples collected during the colonoscopy. Moreover, the inflammation scores obtained from the whole-wall colon and biopsy samples collected during colonoscopy correlated markedly. In conclusion, colonoscopy is a reliable method for following up the course of inflammation in experimentally induced colitis. Although biopsy samples collected during colonoscopies may be used
\end{abstract}

Correspondence to: Professor Magdy El-Salhy, Section for Gastroenterology, Department of Medicine, Stord Helse-Fonna Hospital, Box 4000, Tysevegen 64, 5409 Stord, Norway

E-mail: Magdy.el-salhy@helse-fonna.no

Key words: biopsy, colitis, colonoscopy, rats, trinitrobenzene sulfonic acid to assess the degree of inflammation, whole-wall samples are superior in this regard.

\section{Introduction}

Inflammatory bowel disease (IBD) is comprised of two distinct disorders, ulcerative colitis (UC) and Crohn's disease (CD), which exhibit independent clinical features and are fairly distinct in their organ specificity and histopathological characteristics. Their etiologies are unknown. In the majority of cases, the onset of IBD occurs at a young age during the most productive phase of life $(1,2)$. Anticipation, the development of the disease at an earlier age in offspring than in their parents, has been discussed, but remains controversial (3-7). UC and $\mathrm{CD}$ are chronic diseases, whose clinical courses differ considerably, with frequent relapses or chronically active disease in certain patients, whereas others have years of virtually complete remission $(1,2)$.

IBD remains a major clinical and therapeutic challenge and animal models are required for research into the complexity of the condition and to test the efficacy of anti-inflammatory agents. There are several animal models for IBD, including chemically induced colitis and mutant mice, such as interleukin (IL)-2 and IL-10 knockout mice $(8,9)$. No model reproduces human IBD perfectly, but these models have provided valuable information applicable to human IBD $(8,9)$. Trinitrobenzene sulfonic acid (TNBS) may be used to induce colitis in experimental animals, yielding clinical and morphological features that closely mimic those of Crohn's disease, with the peak of inflammation occurring 2-3 days following the induction of colitis and continuing for a duration of 8 weeks $(8,10,11)$.

It has been demonstrated that the total colonoscopy of a rat is feasible and that the endoscopic inflammation scores correlate well with those obtained using macroscopic and microscopic assessments $(11,12)$. Colonic biopsies may also be obtained successfully during this procedure (12). However, it is unclear as to whether small tissue samples obtained in biopsies, together with a colonoscopy, are sufficient in order to follow the course of the inflammatory processes in experimental animals. Therefore, the present study was undertaken in order to determine the reliability of following 
the inflammatory course of TNBS-induced colitis using a colonoscopy together with biopsy samples obtained during the procedure.

\section{Materials and methods}

Rats. In the present study, we used 20 adult male Wistar rats (Hannover GALASTM; Taconic Farms, Inc., Lille Skensved, Denmark), with a mean weight of $201.9 \mathrm{~g}$ (range 166-246 g). The rats were housed singly in Makrolon III cages and fed a standard diet ad libitum (B\&K Universal AS, Nittedal, Norway), consisting of cereal products $(88.5 \%)$, soy protein $(6 \%)$, animal protein $(2.5 \%)$ and soy oil $(0.5 \%)$, supplemented with vitamins, minerals and amino acids $(2.5 \%)$. Water was also provided ad libitum. The rats were maintained at $21 \pm 1^{\circ} \mathrm{C}$, in a relative humidity of $55 \pm 5 \%$ with a $12 / 12 \mathrm{~h}$ light/dark cycle. The rats were left in the animal house for at least seven days to allow them to acclimatize prior to the experiment. When the rats were fasting, a grid floor was used so that their feces would fall down, thus preventing the rats from eating them. The rats were randomized into two groups, control and TNBS, with ten rats in each. The control group was treated in the same way as the TNBS group, with the exception of the introduction of TNBS intracolonically.

Induction of colitis with TNBS. Rats were deprived of food for $24 \mathrm{~h}$ prior to the administration of TNBS. A single TNBS dose (Sigma-Aldrich Logistik, Steinheim, Germany) was introduced into the colon of each rat $(25 \mathrm{mg} / \mathrm{rat}$ in $50 \%$ ethanol solution; $0.5 \mathrm{ml} / \mathrm{rat}$ ), followed by $2 \mathrm{ml}$ of air, at a distance of $8 \mathrm{~cm}$ from the anal margin via a probe (an $8.5 \mathrm{~cm}$ long, $2.5 \mathrm{~mm}$ round-tip Teflon tube) under isoflurane anesthesia. Following the insertion of TNBS, the rats remained prone with their hind legs raised for at least $1 \mathrm{~min}$. The animals were supervised until recovery and were monitored several times daily. If a rat exhibited signs of pain, it was injected subcutaneously with $0.2 \mathrm{ml} \mathrm{Temgesic}{ }^{\circledR}(0.3 \mathrm{mg}$ Temgesic/ml, Schering-Plough, Whitehouse Station, NJ, USA).

Colonoscopy and mucosal biopsies. Colonoscopy and mucosal biopsies were performed on the control and TNBS rats, three days following the introduction of TNBS in the latter. The bowel was prepared as described previously (12). The rats were fasted for $24 \mathrm{~h}$ and received a gastric dose of 1 and $2 \mathrm{ml}$ Picoprep ${ }^{\circledR}$ (Ferring, West Drayton, UK) followed by $2 \mathrm{ml}$ water at 24 and $12 \mathrm{~h}$, respectively, prior to the colonoscopy. Picoprep consists of a volume of $150 \mathrm{ml}$ containing $10 \mathrm{mg}$ of sodium sulfates, $3.5 \mathrm{~g}$ of magnesium oxide and $12 \mathrm{~g}$ of citric acid. Picoprep was introduced via an $8.5 \mathrm{~cm}$ long, $2.5 \mathrm{~mm}$ round-tip Teflon feeding gauge (AngTheo's AB, Lidingö, Sweden).

The rats were anesthetized by inhalation of isoflurane (Schering-Plough) prior to and during colonoscopy. They were then placed in a supine position and secured to an acrylic surgical table (World Precision Instruments, Sarasota, FL, USA) upon a warming pad (Gaymar T/ Pad; Gaymar Industries, Orchard Park, NY, USA) using a heat therapy pump (TP500 t/Pump; Gaymar) to maintain normothermia during the procedure. A video gastroscope (GIF-N180; Olympus, Tokyo, Japan) was used. Biopsies were obtained with the aid of disposable biopsy forceps (EndoJaw FB-231K; Olympus Medical System, Tokyo, Japan). The tip of the scope was lubricated with $2 \%$ lidocaine (Xylocaine ${ }^{\circledR}$; AstraZeneca, London, UK) and introduced gently into the anus.

The endoscopic grading scale of inflammation used was the same as that used by Vermeulen et al (11). This scale comprises the following five subscales (with a total score ranging from 0-19): degree of inflammation (0-6), extent of disease (0-10), stenosis (0-1), edema (0-1) and active bleeding (0-1).

Following the procedure, the rats were allowed to recover from anesthesia and were monitored for $\sim 1 \mathrm{~h}$. They were then sacrificed by inhalation of $\mathrm{CO}_{2}$ and a post-mortem laparotomy was performed in which the abdomen and colon were examined. The colon was opened and washed with saline solution and the degree of inflammation was assessed using an adaptation of the Wallace and Keenan macroscopic scoring system (13). Using this scoring system, inflammation is assessed on a scale from $0-10$ based on the degree of ulceration, inflammation and extent of disease: 0 , normal aspect of the mucosa; 1, localized hyperaemia without ulcerations; 2, ulceration; 3 , ulceration with thickening of bowel wall at one site; 4, two or more sites of ulceration and thickening of the bowel wall; 5, major sites of damage extending $>1 \mathrm{~cm}$ along the length of the colon; 6-10, damage extending $>2 \mathrm{~cm}$ (the score increases by one for each centimeter of damaged tissue). Tissue samples from the colon were collected for histological examination.

Histopathological examination. Biopsy samples obtained during colonoscopy and whole-wall colon tissues removed during the post-mortem laparotomy were fixed in $4 \%$ buffered paraformaldehyde overnight, embedded in paraffin and cut into $5 \mu \mathrm{m}$ sections. The sections were HE stained. Inflammation was evaluated using the scoring system of Hunter et al (14), in which the total score is a summation of four parameters: inflammatory infiltration (0-3), the number of gut walls engaged (0-3), damage to the mucosal architecture (0-1) and edema (0-1). For biopsy specimens, the number of gut wall engagements was graded 0-2. The total score of this scale ranged from 0-10 for whole-wall colon tissue and 0-9 for the biopsies.

This study was performed in accordance with the Directive for the Protection of Vertebrate Animals used for Experimental and other Scientific Purposes (86/609/EEC), in compliance with the Helsinki Declaration. The local ethical committee for experimental animals at the University of Bergen approved the protocols of the study.

Statistical analysis. The paired t-test and non-parametric Spearman's correlation test were used. $\mathrm{P}<0.5$ was considered to indicate a statistically significant difference. The data are expressed as the means \pm SEM values.

\section{Results}

Prior to the endpoint of the experiment, three rats in the TNBS group died. Post-mortem macroscopic examination of the abdomen and colon of these rats revealed severe colonic inflammation with perforation. Total colonoscopy was 


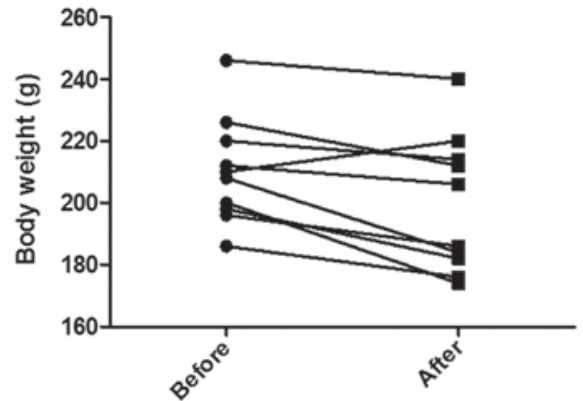

Figure 1. Rat body weights prior to, and three days following, the induction of colitis by TNBS. TNBS, trinitrobenzene sulfonic acid.

A

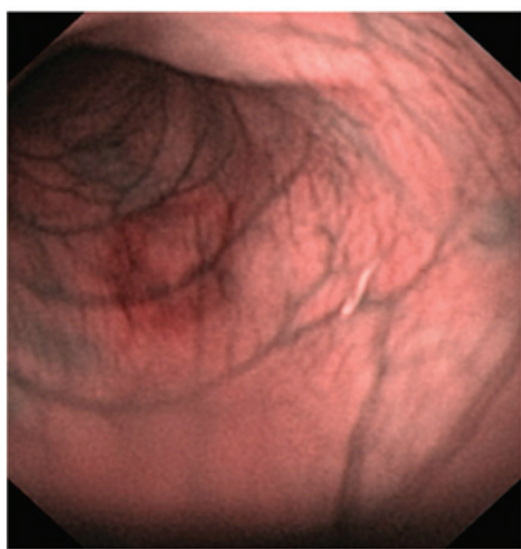

B

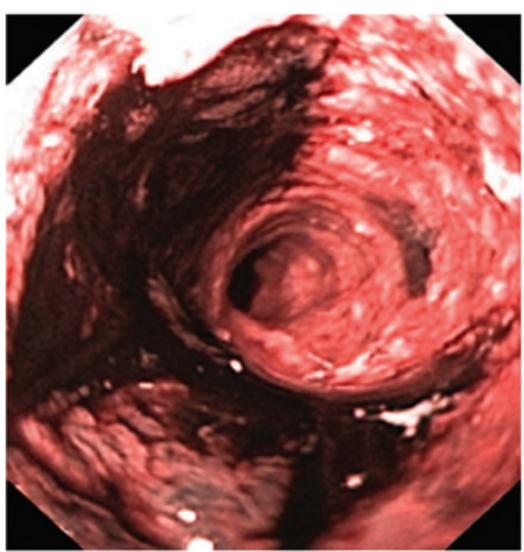

Figure 2. Appearance of the colonic mucosa of (A) a control rat and (B) a rat with TNBS-induced colitis. Narrow-band imaging was used, which provides twice the viewable distance and offers a greater contrast between blood vessels and mucosa. TNBS, trinitrobenzene sulfonic acid.

performed successfully in the remaining rats, who recovered well from the procedure.

The body weight in the control group was $191.5 \pm 9.8 \mathrm{~g}$ at the beginning and end of the experiment. In the TNBS group, the body weight was $210.2 \pm 5.5 \mathrm{~g}$ at the start of the experiment and 199.4 $\pm 7.0 \mathrm{~g}$ at its end point (Fig. 1). This weight loss was considered to be statistically significant $(\mathrm{P}=0.009)$.

Colonoscopy findings. Colonoscopy examinations of the control group did not reveal any inflammation. Severe colitis was observed in every TNBS-induced colitis rat, with a Vermeulen inflammation score of $12.2 \pm 1.0$ (Fig. 2).

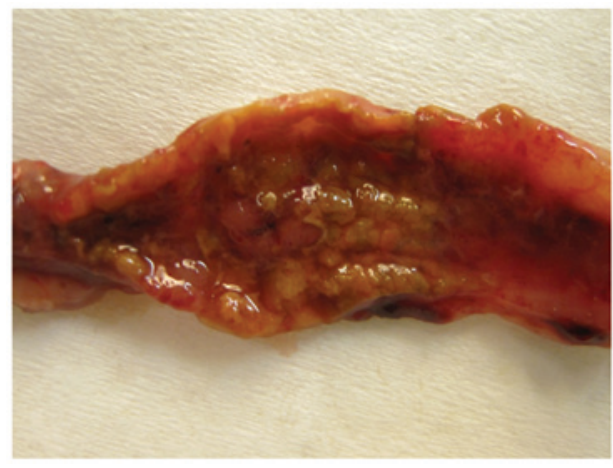

Figure 3. Macroscopic appearance of the colon in a rat with TNBS-induced colitis. TNBS, trinitrobenzene sulfonic acid.

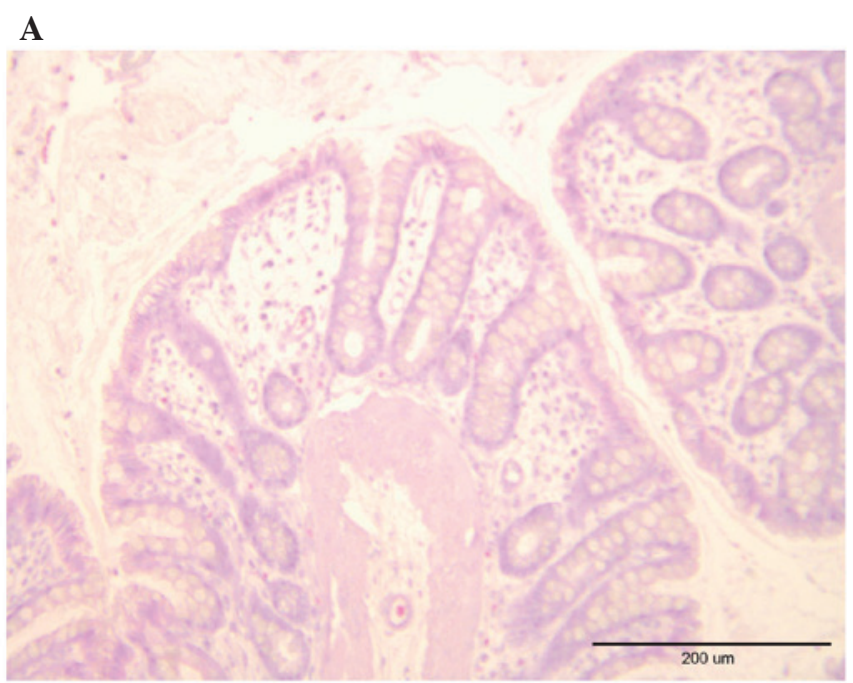

B

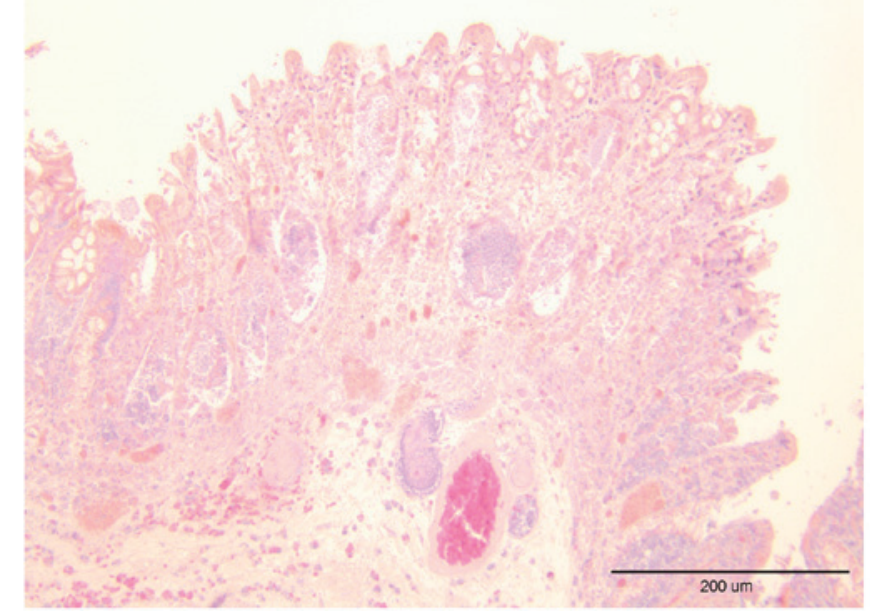

Figure 4. Histopathological changes in the colon in rats three days following the induction of TNBS-colitis in a whole-wall sample of (A) a control rat, and (B) a rat with colitis revealing a typical aphthoid ulcer with a small focus of mucosal damage over the remains of the lymphoid follicle. TNBS, trinitrobenzene sulfonic acid.

Macroscopic appearance. Post-mortem macroscopic examination of the abdomens and colons of the control group did not reveal any complications or inflammation in the colon. The Wallace and Keenan inflammation score for the TNBS group was $5.7 \pm 0.4$ (Fig. 3). 
$\mathbf{A}$

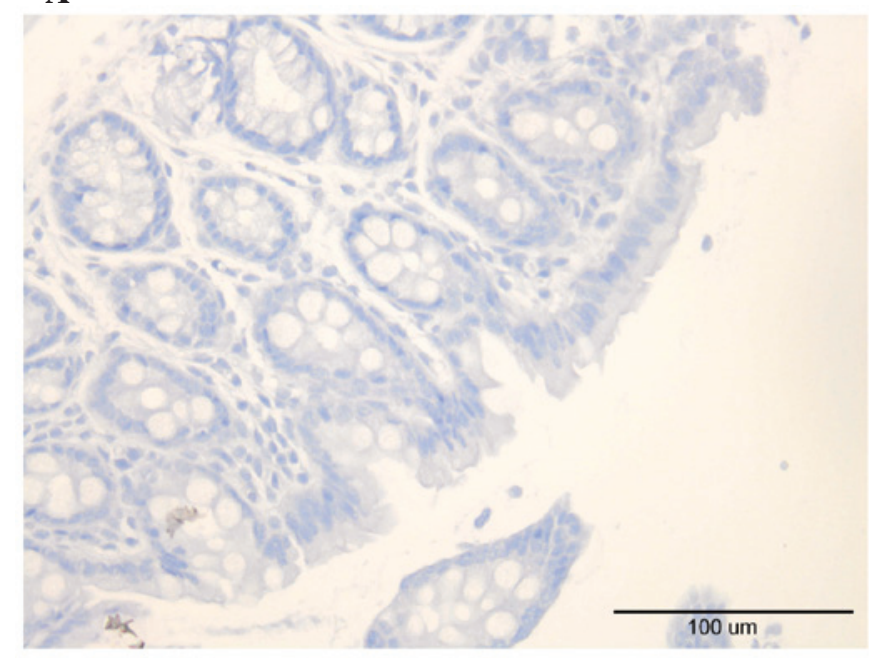

B

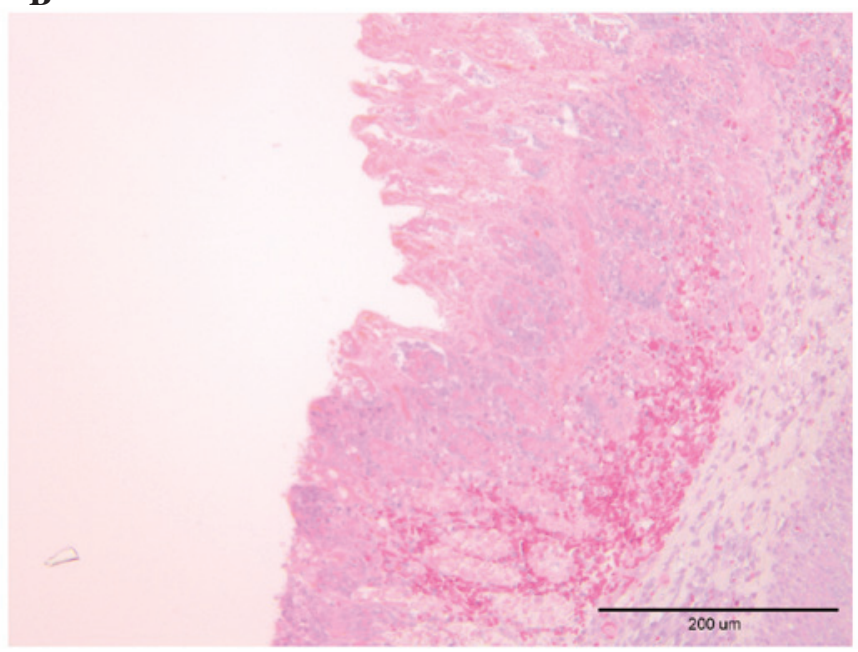

Figure 5. Photomicrographs of colonic biopsy samples obtained during colonoscopy in (A) a control rat and (B) a rat with TNBS-induced colitis. TNBS, trinitrobenzene sulfonic acid.

Histopathological findings. Examination of the whole-wall colonic tissue samples from the control group did not reveal any significant signs of inflammation. The same was true for biopsies obtained during colonoscopy examination. In rats with TNBS-induced colitis, the inflammation scores in whole-wall colonic samples and biopsies collected during colonoscopy

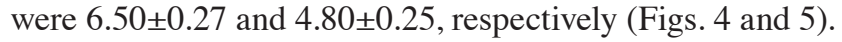

The endoscopic inflammation score obtained through colonoscopy examinations correlated with that established macroscopically $(\mathrm{P}<0.0001, \mathrm{r}=0.9)$, and those obtained microscopically from the score obtained from the whole-wall colonic $(\mathrm{P}=0.01, \mathrm{r}=0.8)$ and biopsy samples collected during colonoscopy $(\mathrm{P}=0.02, \mathrm{r}=0.8)$. The inflammation scores from whole-wall colonic and biopsy samples collected during colonoscopy correlated markedly ( $\mathrm{P}=0.004, \mathrm{r}=0.9$; Fig. 6).

\section{Discussion}

Crohn's disease exhibits endoscopically deep aphthoid ulcerations on otherwise normal mucosa, edema, and fibrotic and inflammatory stenoses (15). Histopathologically, trans-
A

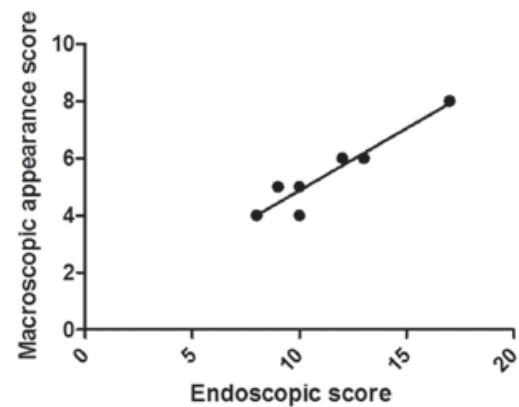

B

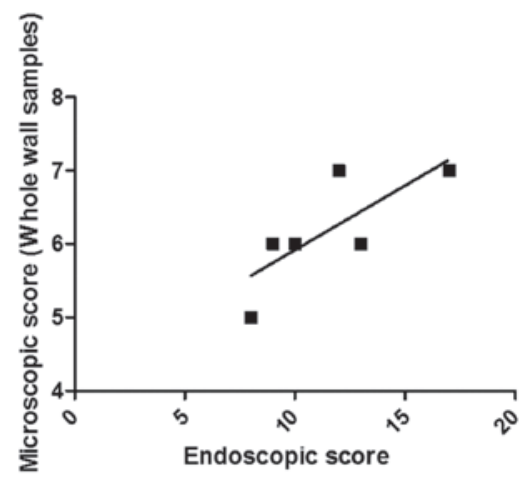

C

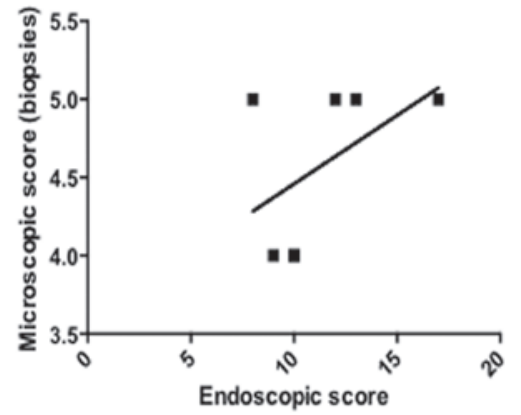

D

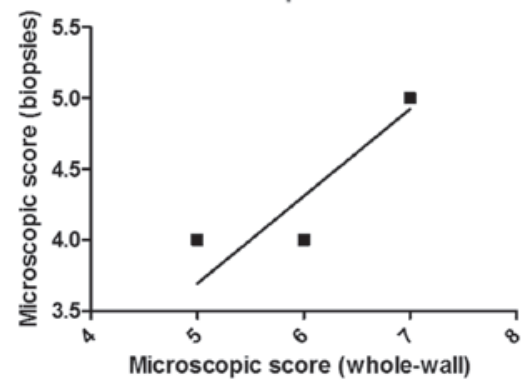

Figure 6. Correlation between the inflammatory scores assessed by colonoscopy, macroscopic appearance, histological assessment in whole-wall samples and biopsy samples obtained during colonoscopy, in addition to the correlation between the inflammatory scores obtained from the whole-wall and biopsy samples.

mural inflammatory ulceration and aphthoid ulceration [small foci with superficial ulcerations deep in the crypts over a lymphoid follicle (16)]. Colonoscopy examination of rats following the induction of TNBS-induced colitis revealed deep widespread ulcers, edema and inflammatory stenoses. The corresponding histopathological findings indicated transmural ulceration, inflammatory cell infiltration and mucosal damage. These findings emphasize the similarities between TNBS-induced colitis and Crohn's disease.

The inflammation was evaluated three days following the induction of colitis. There was a significant weight loss and the inflammatory scores were $64.2,57,65$ and $53.3 \%$ of 
the maximum score as assessed by endoscopy, macroscopic appearance and the histological examination of whole-wall colonic and biopsy samples, respectively. As three rats died due to severe inflammation and perforation, the inflammation that occurred at the time of evaluation should be considered as severe. These findings are in agreement with earlier observations regarding TNBS-induced colitis in rats, of which revealed the maximal inflammation 2-3 days following induction $(8,14)$.

The degree of inflammation, as assessed by endoscopic examinations, correlated markedly with that assessed macroscopically and with the histopathological examination of the colonic whole-wall and biopsy samples obtained during endoscopy. Thus, colonoscopy may be used to follow the inflammatory course in rats with induced colitis, in accordance with previously reported observations (14). Furthermore, the present study revealed that biopsy samples collected during colonoscopy are useful in assessing the degree of inflammation in addition to endoscopic findings. However, the degree of inflammation assessed by endoscopy, expressed as a percentage of the maximum score, is more consistent with the histopathological assessment of whole-wall colonic samples than with the biopsy samples obtained during colonoscopy. This may be due to the fact that i) the whole-wall samples provide a larger area for examination, which is important in TNBS-induced colitis for the spread of pathological changes in otherwise normal mucosa, and/or ii) biopsy samples only include two of the wall layers, namely the mucosa and submucosa, whereas whole-wall samples include a third layer, the muscularis, which is particularly important as the severity of inflammation is dependent upon the depth of ulceration.

In conclusion, colonoscopy provides a reliable method for following up on the course of inflammation in experimentally induced colitis. Although biopsy samples obtained during colonoscopy may be used as well in the assessment of the degree of inflammation, whole-wall samples are superior in this regard.

\section{Acknowledgements}

This study was supported by a grant from Helse-Fonna.

\section{References}

1. Danese S and Fiocchi C: Etiopathogenesis of inflammatory bowel diseases. World J Gastroenterol 12: 4807-4812, 2006.

2. Nunes T, Fiorino G, Danese S and Sans M: Familial aggregation in inflammatory bowel disease: Is it genes or environment? World J Gastroenterol 17: 2715-2722, 2011.

3. Heresbach D, Gulwani-Akolkar B, Lesser M, et al: Anticipation in Crohn's disease may be influenced by gender and ethnicity of the transmitting parent. Am J Gastroenterol 93: 2368-2372, 1998.

4. Grandbastien B, Peeters M, Franchimont D, et al: Anticipation in familial Crohn's disease. Gut 42: 170-174, 1998.

5. Lee JC, Bridger S, McGregor C, Macpherson AJ and Jones JE: Why children with inflammatory bowel disease are diagnosed at a younger age than their affected parent. Gut 44: 808-811, 1999.

6. Faybush EM, Blanchard JF, Rawsthorne P and Bernstein CN: Generational differences in the age at diagnosis with Ibd: genetic anticipation, bias, or temporal effects. Am J Gastroenterol 97: 636-640, 2002

7. Hampe J, Heymann K, Kruis W, Raedler A, Fölsch UR and Schreiber S: Anticipation in inflammatory bowel disease: a phenomenon caused by an accumulation of confounders. Am J Med Genet 92: 178-183, 2000.

8. Elson CO, Sartor RB, Tennyson GS and Riddell RH: Experimental models of inflammatory bowel disease. Gastroenterology 109: 1344-1367, 1995.

9. Saleh $\mathrm{M}$ and Elson CO: Experimental inflammatory bowel disease: insights into the host-microbiota dialog. Immunity 34 : 293-302, 2011.

10. Milde AM and Murison R: A study of the effects of restraint stress on colitis induced by dextran sulphate sodium in singly housed rats. Integr Physiol Behav Sci 37: 140-150, 2002.

11. Vermeulen W, De Man JG, Nullens S, Pelckmans PA, De Winter BY and Moreels TG: The use of colonoscopy to follow the inflammatory time course of TNBS colitis in rats. Acta Gastroenterol Belg 74: 304-311, 2011.

12. El-Salhy M, Wendelbo I, Gundersen D, Hatlebakk JG and Husken T: Colonoscopy in young rats with mucosa biopsies: A model for experimental gastroenterology. Mol Med Rep 7: 1757-1760, 2013.

13. Wallace JL and Keenan CM: An orally active inhibitor of leukotriene synthesis accelerates healing in a rat model of colitis. Am J Physiol 258: G527-G534, 1990.

14. Hunter MM, Wang A, Hirota CL and McKay DM: Neutralizing anti-IL-10 antibody blocks the protective effect of tapeworm infection in a murine model of chemically induced colitis. J Immunol 174: 7368-7375, 2005.

15. Modigliani R: Endoscopy. In: Inflammatory bowel disease. Järnerot G, Lennard-Jones J and Truelove S (eds.) Corona Astra, Malmö, Sweden, pp 243-267, 1992.

16. Modigliani R: The pathology. In: Inflammatory bowel disease. Järnerot G, Lennard-Jones J and Truelove S (eds.) Corona Astra, Malmö, Sweden, pp 269-293, 1992. 